\title{
Health Status among Elderly in One Region of Thailand
}

\author{
Rujira Jeamamornrat, MSN. ${ }^{1,}$ Chularat Howharn PhD. ${ }^{2}$ \\ ${ }^{1}$ Praboromarajchanok Institute ${ }^{2}$ Boromarajonani College of Nursing, Surin
}

\begin{abstract}
Background: In order to promote aging to modify their lifestyle for enhance their health status, information related to their activities of daily living (ADL) should be in-depth describe. Information are values for develop intervention with the aim to maintain and extend their good health status; especially for the setting that have different index of aging (IOA).

Objectives: The aims of this study were to describe activity of daily living in elderly among two group of index of aging, One Region of Thailand.

Methodology: This study was a descriptive study. Setting were 6 provinces in One Region of Thailand and one-two village was simple random from province with the IOA between 60.79.9 and two villages were simple random from province with the IOA between 80-99.9. Total were 8 villages. 1,609 cases were purposive selected from elderly who were age from 60 years old. Data were collected by geriatric screening form which was developed by Elderly Medicine Institute. Descriptive study was used to describe the findings.

Results: The finding revealed that mean age was $70.59($ SD. $=7.59)$ and $61.8 \%$ were female. Most of elderly were well elder $(97.20 \%)$ with $2.10 \%$ were home bound and were $0.70 \%$ were bed bound. The mean score of ADL for lower IOA province was 19.32 while the mean score for higher IOA province was 18.67 with the statistically different at $\mathrm{p}<.05$.

Conclusion and Recommendation: The findings revealed that the older the higher score of ADL. The higher IOA the lower score of ADL; therefore, any intervention with the aims to promote their good health study should be developed; especially for the home bound group in province with the higher IOA. Moreover, since the major of elderly have a good connection with the communities, therefore social capitol should be integrated into that intervention.
\end{abstract}

Keywords: activity of daily living, elderly, healthy aging 